\title{
Late-time Inhomogeneity and Acceleration of the Universe
}

\author{
J. W. Moffat \\ The Perimeter Institute for Theoretical Physics, Waterloo, Ontario, N2J 2W9, \\ Canada \\ and \\ Department of Physics, University of Waterloo, Waterloo, Ontario N2L 3G1, \\ Canada
}

\begin{abstract}
The inhomogeneous distribution of matter in the non-linear regime of galaxies, clusters of galaxies and voids is described by an exact, spherically symmetric inhomogeneous solution of Einstein's gravitational field equations, corresponding to an under-dense void. The solution becomes the homogeneous and isotropic Einstein-de Sitter solution for a red shift $z>10-20$, which describes the matter dominated CMB data with small inhomogeneities. A spatial volume averaging of physical quantities is introduced and the averaged time evolution expansion parameter $\theta$ can give rise in the late-time universe to a volume averaged deceleration parameter $\langle q\rangle$ that is negative for a positive matter density. This allows for a region of accelerated expansion which does not require a cosmological constant. A negative deceleration parameter can be derived by this volume averaging procedure from the Lemaitre-Tolman-Bondi open void solution, which describes the late-time non-linear regime associated with galaxies and under-dense voids and solves the "coincidence" problem.
\end{abstract}

email: john.moffat@perimeterinstitute.ca

\section{Introduction}

In recent articles, we investigated a cosmology in which a spherically symmetric inhomogeneous enhancement is embedded in an asymptotic FLRW universe 1, 2..$^{1}$ The inhomogeneous enhancement is described by an exact inhomogeneous solution of Einstein's field equations. The inhomogeneous distribution of matter at late times can possibly allow for a negative deceleration parameter $q$, depending on the nature of the exact inhomogeneous solution of Einstein's field equations. This will allow for the possibility of explaining the acceleration of the universe, without a cosmological constant. The model also leads to an

\footnotetext{
${ }^{1}$ For a more complete list of references, see refs. 1$] 2$
} 
axis pointing towards the center of a spherically symmetric inhomogeneous enhancement with dipole, quadrupole and octopole moments aligned with the axis. The distribution of CMB temperature fluctuations can be unevenly distributed in the northern and southern hemispheres [1.

We shall describe the inhomogeneous late-time universe by an exact spherically symmetric solution of Einstein's field equations. The solution becomes the homogeneous and isotropic Einstein-de Sitter matter dominated solution for $z>20$, so that it can describe the WMAP data near the surface of last scattering with small temperature perturbations $\delta T / T \sim 10^{-5}[3$. However, for $z<20$ the solution is inhomogeneous and for luminosity distances corresponding to $z \sim 0.2-1.4$, includes the Type SNIa supernova measurements 4, [5]. We investigate the expansion geometry of the spherical distribution of inhomogeneous matter and under-dense voids $[\underline{6}$ to see whether it allows for the possibility of a negative $q$ and an accelerating expansion of the universe, without negative pressure dark energy or a cosmological constant.

A generic relativistic solution of a three-dimensional inhomogeneous universe is presently unknown. The highly symmetric Lemaitre-Tolman-Bondi (LTB) [7, 8. 9] solution that we shall consider in the following is inhomogeneous in only one spatial dimension. Even though the assumption of spherical symmetry is unrealistic, the model is useful in illustrating important local features of physical quantities.

For our inhomogeneous late-time universe model, we are required to carry out a volume averaging of physical scalar quantities, such as the expansion parameter $\theta$. We find for such an averaging process that for a zero cosmological constant and for irrotational matter the average deceleration parameter $\langle q\rangle$ can be negative and describe an accelerating local region of the universe, corresponding to our observed Hubble radius, without invoking a cosmological constant.

\section{Inhomogeneous Friedmann Equations}

For the sake of notational clarity, we write the FLRW line element

$$
d s^{2}=d t^{2}-a^{2}(t)\left(\frac{d r^{2}}{1-k r^{2}}+r^{2} d \Omega^{2}\right),
$$

where $k=+1,0,-1$ for a closed, flat and open universe, respectively, and $d \Omega^{2}=d \theta^{2}+\sin \theta^{2} d \phi^{2}$. The general, spherically symmetric inhomogeneous line element is given by [7, 8, 9, 10, 11, 12, 13, 1]:

$$
d s^{2}=d t^{2}-X^{2}(r, t) d r^{2}-R^{2}(r, t) d \Omega^{2} .
$$

The energy-momentum tensor $T^{\mu}{ }_{\nu}$ takes the barytropic form

$$
T^{\mu}{ }_{\nu}=(\rho+p) u^{\mu} u_{\nu}-p \delta^{\mu}{ }_{\nu},
$$


where $u^{\mu}=d x^{\mu} / d s$ and, in general, the density $\rho=\rho(r, t)$ and the pressure $p=p(r, t)$ depend on both $r$ and $t$. We have for comoving coordinates $u^{0}=$ $1, u^{i}=0,(i=1,2,3)$ and $g^{\mu \nu} u_{\mu} u_{\nu}=1$.

The Einstein gravitational equations are

$$
G_{\mu \nu}+\Lambda g_{\mu \nu}=-8 \pi G T_{\mu \nu}
$$

where $G_{\mu \nu}=R_{\mu \nu}-\frac{1}{2} g_{\mu \nu} \mathcal{R}, \mathcal{R}=g^{\mu \nu} R_{\mu \nu}$ and $\Lambda$ is the cosmological constant. Solving the $G_{01}=0$ equation for the metric (2), we find that

$$
X(r, t)=\frac{R^{\prime}(r, t)}{f(r)}
$$

where $R^{\prime}=\partial R / \partial r$ and $f(r)$ is an arbitrary function of $r$.

We obtain the two generalized Friedmann equations [1]:

$$
\begin{gathered}
\frac{\dot{R}^{2}}{R^{2}}+2 \frac{\dot{R}^{\prime}}{R^{\prime}} \frac{\dot{R}}{R}+\frac{1}{R^{2}}\left(1-f^{2}\right)-2 \frac{f f^{\prime}}{R^{\prime} R}=8 \pi G \rho+\Lambda, \\
\frac{\ddot{R}}{R}+\frac{1}{3} \frac{\dot{R}^{2}}{R^{2}}+\frac{1}{3} \frac{1}{R^{2}}\left(1-f^{2}\right)-\frac{1}{3} \frac{\dot{R}^{\prime}}{R^{\prime}} \frac{\dot{R}}{R}+\frac{1}{3} \frac{f f^{\prime}}{R^{\prime} R}=-\frac{4 \pi G}{3}(\rho+3 p)+\frac{1}{3} \Lambda,
\end{gathered}
$$

where $\dot{R}=\partial R / \partial t$.

\section{Late-Time Matter Dominated Universe}

The late-time matter dominated universe will be pictured as a large-scale inhomogeneous enhancement that is described by an exact inhomogeneous spherically symmetric solution of Einstein's field equations. The inhomogeneous enhancement is embedded in a matter dominated universe that approaches asymptotically an Einstein-de Sitter universe as $t \rightarrow \infty$. An observer will be off-center from the origin of coordinates of the spherical inhomogeneous enhancement. The local inhomogeneous solution corresponds to an open, hyperbolic solution and describes an under-dense void. Surveys such as the 2-degree Field Galaxy Redshift Survey and the Sloan Digital Sky Survey show large volumes of relatively empty space, or voids, in the distribution of galaxies [6.

For the matter dominated Lemaitre-Tolman-Bondi (LTB) [7, 8, 9] model with zero pressure $p=0$ and zero cosmological constant $\Lambda=0$, the Einstein field equations demand that $R(r, t)$ satisfies

$$
2 R(r, t) \dot{R}^{2}(r, t)+2 R(r, t)\left(1-f^{2}(r)\right)=F(r),
$$

with $F$ being an arbitrary function of $r$ of class $C^{2}$.

The proper density of matter can be expressed as

$$
\rho=\frac{F^{\prime}}{16 \pi G R^{\prime} R^{2}} .
$$


We can solve (9) to obtain

$$
\Omega-1 \equiv \frac{\rho}{\rho_{c}}-1=\frac{1}{H_{\mathrm{eff}}^{2}}\left(\frac{1-f^{2}}{R^{2}}-2 \frac{f}{R} \frac{f^{\prime}}{R^{\prime}}\right),
$$

where

$$
H_{\text {eff }}^{2}=H_{\perp}^{2}+2 H_{\perp} H_{r},
$$

is an effective Hubble parameter and

$$
H_{r}=\frac{\dot{R}^{\prime}}{R^{\prime}}, \quad H_{\perp}=\frac{\dot{R}}{R} .
$$

We have for the critical density

$$
8 \pi G \rho_{c}=H_{\mathrm{eff}}^{2} .
$$

There are three possibilities for the curvature of spacetime: 1) $f^{2}>1$ open $(\Omega-1<0), 2) f^{2}=1$ flat $(\Omega-1=0), f^{2}<1$ closed $(\Omega-1>0)$.

We describe the inhomogeneous density regime by a hyperbolic, open solution $f^{2}(r)>1$ with $p=\Lambda=0$, corresponding to an under-dense void. We choose

$$
f(r)=\sqrt{1+r^{2}}
$$

and the metric reduces to

$$
d s^{2}=d t^{2}-\frac{R^{\prime 2}(r, t)}{1+r^{2}} d r^{2}-R^{2}(r, t) d \Omega^{2} .
$$

A parametric solution is given by

$$
\begin{aligned}
R(r, t) & =\frac{1}{4} F(r)\left(f^{2}(r)-1\right)^{-1}(\cosh u(r, t)-1), \\
t+\beta(r) & =\frac{1}{4}\left(f^{2}(r)-1\right)^{-3 / 2}(\sinh u(r, t)-u(r, t)), \\
8 \pi G \rho(r, t) & =\left(\frac{2 F^{\prime}(r)}{F^{2}(r)}\right)\left(\frac{f^{2}(r)-1}{R^{\prime}(r, t)}\right)\left(\frac{1}{\sinh ^{4} \frac{1}{2} u(r, t)}\right) .
\end{aligned}
$$

Here, $\beta(r)$ is an arbitrary function of $r$ of class $C^{2}[10$.

The function $\beta(r)$ can be specified in terms of a density on some spacelike hypersurface $t=t_{0}$. The metric and density are singular for

$$
u=0 \quad \text { or } \quad R^{\prime}(r, t)=0,
$$

where $u=0$ refers to the hypersurface $t+\beta(r)=0$ and the singular surface for $R^{\prime}(r, t)=0$ is more complicated. Our pressureless model requires that the singular surface $t(r)=\Sigma(r)$ describes the surface on which the universe becomes matter dominated (in the FLRW model this occurs at $z \sim 10^{4}$ ). The hyperbolic FLRW model is obtained by choosing the conditions

$$
f^{2}(r)=1+r^{2}, \quad F(r)=4 s r^{3}, \quad \beta(r)=0,
$$


where $s$ is a positive constant. This leads to the metric solution

$$
\begin{gathered}
R=s r(\cosh u-1), \\
t=s(\sinh u-u), \\
8 \pi G \rho=\frac{3}{4 s^{2}}\left(\frac{1}{\sinh ^{6} \frac{1}{2} u}\right) .
\end{gathered}
$$

We shall change the radial coordinate to

$$
r^{*}=r-r_{S L S},
$$

where $r=r_{S L S}$ is the location of the surface of last scattering corresponding to $r^{*}=0$ at a red shift $z \sim 1100$. We postulate that $\beta\left(r^{*}\right)$ approaches a maximum constant value at the approximate end of galaxy and cluster formation, $z_{f} \sim$ $1-5$, where $z=z_{f}$ denotes the final value of the red shift. We assume that $\beta\left(r^{*}\right) \rightarrow 0$ and $f\left(r^{*}\right) \rightarrow 1$ as $r^{*} \rightarrow 0$. A model for $\beta$ is of the form:

$$
\beta\left(r^{*}\right)=a\left(\frac{r^{*}}{b+r^{*}}\right)^{\alpha}
$$

where $a, b$ and $\alpha$ are positive constants, $\beta(0)=0$ and $\beta\left(r^{*}\right) \rightarrow a$ for $r^{*} \gg b$. The surface $r^{*}=0$ for the metric is a singular surface.

The metric for $f\left(r^{*}\right) \rightarrow 1$ becomes [10]:

$$
d s^{2}=d t^{2}-\left(t+\beta\left(r^{*}\right)\right)^{4 / 3}\left(Y^{2}\left(r^{*}, t\right) d r^{* 2}+r^{* 2} d \Omega^{2}\right),
$$

where

$$
Y\left(r^{*}, t\right)=1+\frac{2 r^{*} \beta^{\prime}\left(r^{*}\right)}{3\left(t+\beta\left(r^{*}\right)\right)}
$$

and

$$
\rho\left(r^{*}, t\right)=\frac{1}{6 \pi G\left(t+\beta\left(r^{*}\right)\right)^{2} Y\left(r^{*}, t\right)} .
$$

We obtain for $\beta\left(r^{*}\right) \rightarrow 0$ the homogeneous and isotropic solution corresponding to a spatially flat Einstein-de Sitter universe with the metric

$$
d s^{2}=d t^{2}-a^{2}(t)\left(d r^{* 2}+r^{* 2} d \Omega^{2}\right),
$$

and $a(t) \propto t^{2 / 3}$. This solution is compatible as a background spacetime with the approximately homogeneous and isotropic WMAP data for $20<z<10^{3}$ and $\delta \rho / \rho \sim 10^{-5}[3]$.

The Einstein-de Sitter solution must be supplemented by non-zero pressure contributions for $z \sim 10^{3}$ in the linear regime at the surface of last scattering in order to take into account the radiation density $\rho_{R}$. Moreover, a more general solution is required that has a non-vanishing cosmological constant $\Lambda$, so that we have $\Omega=\Omega_{M}+\Omega_{R}+\Omega_{\Lambda}=1$ in agreement with the spatially flat result $\Omega=1.02 \pm 0.02$, obtained from the determination of the first acoustic peak in the WMAP power spectrum [3]. However, in the following section, we shall replace $\Omega_{\Lambda}$ by a contribution corresponding to an acceleration parameter $q$ obtained from our inhomogeneous void solution with $\Lambda=0$. 


\section{The Inhomogeneous Late-Time Density and Deceleration Parameters}

We obtain from (6) for $f(r)=\sqrt{1+r^{2}}$ and $p=0$ (we replace for convenience $r^{*}$ by $\left.r\right)$ :

$$
H_{\perp}^{2}+2 H_{r} H_{\perp}-\frac{r^{2}}{R^{2}}-\frac{2 r}{R^{\prime} R}=8 \pi G \rho+\Lambda .
$$

Dividing this equation by $H_{\perp}^{2}$ gives

$$
\Omega \equiv \frac{8 \pi G \rho}{H_{\perp}^{2}}+\frac{\Lambda}{H_{\perp}^{2}}+\frac{r^{2}}{R^{2} H_{\perp}^{2}}+\frac{2 r}{R^{\prime} R H_{\perp}^{2}}-\frac{2 H_{r}}{H_{\perp}}=1 .
$$

Let us expand $R(r, t)$ in a Taylor series

$$
\begin{gathered}
R(r, t)=R\left[r, t_{0}-\left(t_{0}-t\right)\right]=R\left(r, t_{0}\right)\left[1-\left(t_{0}-t\right) \frac{\dot{R}\left(r, t_{0}\right)}{R\left(r, t_{0}\right)}+\frac{1}{2}\left(t_{0}-t\right)^{2} \frac{\ddot{R}\left(r, t_{0}\right)}{R\left(r, t_{0}\right)}-\ldots\right] \\
=R\left(r, t_{0}\right)\left[1-\left(t_{0}-t\right) H_{0 \perp}-\frac{1}{2}\left(t_{0}-t\right)^{2} q\left(r, t_{0}\right) H_{0 \perp}^{2}-\ldots\right]
\end{gathered}
$$

where $t_{0}$ denotes the present epoch and $H_{0 \perp}=\dot{R}\left(r, t_{0}\right) / R\left(r, t_{0}\right)$. Moreover, we have

$$
q\left(r, t_{0}\right)=-\frac{\ddot{R}\left(r, t_{0}\right) R\left(r, t_{0}\right)}{\dot{R}^{2}\left(r, t_{0}\right)} .
$$

For the matter dominated era we set $p=0$ in Eqs. (6) and (7) and substitute for $\ddot{R}$ from (7) to obtain

$$
\begin{gathered}
q\left(r, t_{0}\right)=\frac{1}{3}+\frac{4 \pi \rho_{0}\left(r, t_{0}\right)}{3 H_{0 \perp}^{2}\left(r, t_{0}\right)} \\
+\frac{1}{3} \frac{r}{R R^{\prime} H_{0 \perp}^{2}}-\frac{\Lambda}{3 H_{0 \perp}^{2}\left(r, t_{0}\right)}-\frac{1}{3} \frac{H_{0 r}\left(r, t_{0}\right)}{H_{0 \perp}\left(r, t_{0}\right)}-\frac{1}{3} \frac{r^{2}}{R^{2} H_{0 \perp}^{2}}
\end{gathered}
$$

where $H_{0 r}\left(r, t_{0}\right)=\dot{R}^{\prime}\left(r, t_{0}\right) / R^{\prime}\left(r, t_{0}\right)$.

If we set $H_{0 \perp}\left(r, t_{0}\right)=H_{0 r}\left(r, t_{0}\right)=H\left(t_{0}\right)$ where $H\left(t_{0}\right)=\dot{a}\left(t_{0}\right) / a\left(t_{0}\right)$ and $R\left(r, t_{0}\right)=a\left(t_{0}\right)$, then we obtain the global FLRW expression for the deceleration parameter for a spatially flat universe with $f^{2}(r)=1$ :

$$
q_{0}=\frac{1}{2} \Omega_{0 M}-\Omega_{0 \Lambda}
$$

where from (31) $\Omega_{0 M}+\Omega_{0 \Lambda}=1$ with $\Omega_{0 M}=8 \pi \rho_{0 M} / 3 H^{2}\left(t_{0}\right)$ and $\Omega_{0 \Lambda}=$ $\Lambda / 3 H^{2}\left(t_{0}\right)$.

The variance of $q$ is given by the exact non-perturbative expression:

$$
\operatorname{var}(q) \equiv\left\langle q^{2}-\langle q\rangle^{2}\right\rangle^{1 / 2}=\bar{q}
$$


where

$$
\overline{(\ldots)}=\frac{\int d^{3} x \sqrt{\gamma}(\ldots)}{\int d^{3} x \sqrt{\gamma}}
$$

denotes the ensemble average and $\gamma$ denotes the determinant of the 3 -dimensional metric $g_{i j}(i=1,2,3)$.

We see from (34) that different observers located in different causally disconnected parts of the sky will observe different values for the deceleration parameter $q$, depending upon their location and distance from the center of the spherically symmetric inhomogeneous enhancement. This can lead to one form of cosmic variance, because the spatial average of all the observed values of local physical quantities, including the deceleration parameter, $q$, will have an intrinsic uncertainty.

Let us set the cosmological constant to zero, $\Lambda=0^{2}$. From (31) and (34), we obtain for $t=t_{0}$ :

$$
\Omega_{0} \equiv \frac{8 \pi G \rho_{0}}{H_{0 \perp}^{2}}+\frac{r^{2}}{R^{2} H_{0 \perp}^{2}}+\frac{2 r}{R^{\prime} R H_{0 \perp}^{2}}-\frac{2 H_{0 r}}{H_{0 \perp}}=1
$$

and

$$
q\left(r, t_{0}\right)=\frac{1}{3}+\frac{4 \pi G \rho_{0}\left(r, t_{0}\right)}{3 H_{0 \perp}^{2}\left(r, t_{0}\right)}+\frac{1}{3} \frac{r}{R R^{\prime} H_{0 \perp}^{2}}-\frac{1}{3} \frac{H_{0 r}\left(r, t_{0}\right)}{H_{0 \perp}\left(r, t_{0}\right)}-\frac{1}{3} \frac{r^{2}}{R^{2} H_{0 \perp}^{2}} .
$$

If in (39) we have

$$
H_{0 r} H_{0 \perp}+\frac{r^{2}}{R^{2}}>4 \pi G \rho_{0}+H_{0 \perp}^{2}+\frac{r}{R R^{\prime}},
$$

then the deceleration parameter $q$ can be negative and cause the universe to accelerate without a cosmological constant or dark energy. If we solve for $H_{0 r} / H_{0 \perp}$ from Eq.(38) and substitute into (39), we obtain

$$
q\left(r, t_{0}\right)=\frac{1}{2}-\frac{5}{6}\left(\frac{r^{2}}{R^{2} H_{0 \perp}^{2}}\right)
$$

which gives a negative $q$ for

$$
r^{2} / R^{2} H_{0 \perp}^{2}>3 / 5
$$

and we can satisfy the condition (38). We see that for $r=0$ corresponding to $f^{2}(r)=1$ and a spatially flat universe $q\left(r, t_{0}\right)=1 / 2$. For $p=\Lambda=0$, Eqs.(17) and (40) lead to $\ddot{R}>0$ as the condition for an accelerating expansion of the universe.

The conditions (38) and (40) require that $f^{2}(r)>1$ for the inhomogeneous enhancement, corresponding to an under-dense void. If we choose instead to describe the inhomogeneity by the spatially flat solution $f^{2}(r)=1$, then we

\footnotetext{
${ }^{2}$ We do not provide any solution to the cosmological constant problem, namely, why $\Lambda=0$.
} 
cannot simultaneously satisfy $\Omega=1$ and $q<0$. However, we will investigate in the next Section, whether the condition (40) can indeed be satisfied for the particular LTB model that we have adopted. We shall find that a more general inhomogeneous solution is needed to establish whether such a solution can explain the accelerating expansion of the universe without a negative pressure fluid or a cosmological constant.

\section{The Raychaudhuri Equation}

The Raychaudhuri equation is 14

$$
\frac{d \theta}{d s}=-R_{\mu \nu} V^{\mu} V^{\nu}+2 \omega^{2}-2 \sigma^{2}-\frac{1}{3} \theta^{2},
$$

where we have adopted geodesic world lines, $V^{\mu}$ is a time-like vector and

$$
\omega^{2}=\omega^{\mu \nu} \omega_{\mu \nu} \geq 0, \quad \sigma^{2}=\sigma^{\mu \nu} \sigma_{\mu \nu} \geq 0 .
$$

Here, $\sigma_{\mu \nu}$ and $\omega_{\mu \nu}$ denote the shear tensor and the vorticity tensor, respectively. From Einstein's field equations, we have for $p=\Lambda=0$ :

$$
\frac{d \theta}{d s}=2 \omega^{2}-2 \sigma^{2}-\frac{1}{3} \theta^{2}-4 \pi G \rho .
$$

The deceleration parameter $q$ is defined by

$$
q \equiv-\frac{\left(3 d \theta / d s+\theta^{2}\right)}{\theta^{2}}=\frac{6\left(\sigma^{2}-\omega^{2}\right)+4 \pi G \rho}{\theta^{2}} .
$$

If the vorticity $\omega=0$, then the deceleration parameter $q$ has to be positive for positive $\rho$.

For our synchronous comoving coordinates we have

$$
\frac{d \theta}{d s}=V^{\mu} \nabla_{\mu} \theta=V^{\mu} \partial_{\mu} \theta=\dot{\theta} .
$$

We have

$$
q \equiv-\frac{\left(3 \dot{\theta}+\theta^{2}\right)}{\theta^{2}}=-\frac{\left(3 \frac{\ddot{R}^{\prime}}{R^{\prime}}+6 \frac{\ddot{R}}{R}+\theta^{2}-3 H_{r}^{2}-6 H_{\perp}^{2}\right)}{\theta^{2}} .
$$

We may now conclude that if

$$
3 H_{r}^{2}+6 H_{\perp}^{2}>3 \frac{\ddot{R}^{\prime}}{R^{\prime}}+6 \frac{\ddot{R}}{R}+\theta^{2},
$$

then $q$ could be negative leading to a local acceleration of the universe. However, for our synchronous comoving frame, we have

$$
\omega_{\mu \nu} \equiv \nabla_{[\nu} V_{\mu]}=\partial_{\nu} V_{\mu}-\partial_{\mu} V_{\nu}=0,
$$


so that the vorticity for this chosen gauge is zero. Therefore, we must deduce that the condition (49) cannot be satisfied for the positive energy condition $\rho>0$ and $p>0$. Moreover, the two conditions (40) and (42) cannot be satisfied for the synchronous and comoving metric (2). However, in the following section, we will show that we must carry out a spatial volume averaging of the expansion parameter $\theta$ and its time evolution to arrive at a physically viable description of the local inhomogeneous late-time universe and an accelerating expansion of the universe.

\section{Spatially Averaged Cosmological Domains}

For our inhomogeneous model, it is necessary to perform a spatial average (37) of physical quantities, due to their observer, location dependence. Let us define a more specific domain averaged expression for a scalar quantity [15, 16] 17]:

$$
\langle\Psi(\vec{x}, t)\rangle_{D}=\frac{1}{\mathcal{V}_{\mathcal{D}}} \int_{D} d^{3} x \sqrt{\gamma} \Psi(\vec{x}, t),
$$

where

$$
\mathcal{V}_{D}=\int_{D} d^{3} x \sqrt{\gamma}
$$

is the volume of the simply-connected domain, $D$, in a hypersurface. We can define an effective scale-factor for our spatially averaged spherically symmetric inhomogeneous enhancement (void):

$$
\langle R(r, t)\rangle_{D}=\left(\frac{\mathcal{V}(t)_{D}}{\mathcal{V}_{i D}}\right)^{1 / 3}
$$

where $\mathcal{V}_{i D}$ is the initial volume.

The volume averaging of the scalar $\Psi$ does not commute with its time evolution [15, 16, 17]:

$$
\langle\dot{\Psi}(r, t)\rangle_{D}-\partial_{t}\langle\Psi(r, t)\rangle_{D}=\langle\Psi(r, t)\rangle_{D}\langle\theta(r, t)\rangle_{D}-\langle\Psi(r, t) \theta(r, t)\rangle_{D} .
$$

We can derive for the averaged $\langle\theta\rangle_{D}$ the equation

$$
\langle\theta\rangle_{D}=3 \frac{\langle\dot{R}\rangle_{D}}{\langle R\rangle_{D}}=3\left\langle H_{\perp}\right\rangle_{D} .
$$

Setting $\Psi=\theta$ and substituting for the Raychaudhuri equation for an irrotational matter dominated late-time model with $\omega_{\mu \nu}=0, d \theta / d s=d \theta / d t$ and $\Lambda=0$, we obtain the expression

$$
3 \frac{\langle\ddot{R}\rangle_{D}}{\langle R\rangle_{D}}=-4 \pi G\langle\rho\rangle_{D}+\langle P\rangle_{D},
$$

where $\langle P\rangle_{D}$ is a function of $\langle\theta\rangle_{D}$, the average Hubble expansion parameters $\left\langle H_{\perp}\right\rangle_{D}$ and $\left\langle H_{r}\right\rangle_{D}$ and the average shear $\langle\sigma\rangle_{D}$. 
For inhomogeneous cosmology, the smoothing due to averaging of the Einstein field equations does not commute with the time evolution of the non-linear field equations. This leads to extra contributions in the effective, averaged Einstein field equations, which do not satisfy the usual energy conditions even though they are satisfied by the original energy-momentum tensor. It is the lack of commutativity of the time evolution of the expansion of the universe in a local patch inside our Hubble horizon that circumvents the no-go theorem based on the local Raychaudhuri equation [2].

If we can satisfy the condition

$$
\langle P\rangle_{D}>4 \pi G\langle\rho\rangle_{D},
$$

then the averaged deceleration parameter $\langle q\rangle_{D}$ can be negative corresponding to the acceleration of a local patch of the universe. If we substitute the value of $\langle\ddot{R}\rangle_{D}$ from the volume averaged equation (17) for $\Lambda=0$, then the exact LTB solution for an irrotational inhomogeneous, under-dense void solution can describe the local acceleration of the late-time universe and fulfill the constraint equation (57). The spatial domain average of $q$ in Eq.411) gives

$$
\langle q\rangle_{D}=\frac{1}{2}-\frac{5}{6}\left\langle\left(\frac{r^{2}}{R^{2} H_{0 \perp}^{2}}\right)\right\rangle_{D} .
$$

This yields the averaged condition for an accelerating universe:

$$
\left\langle\frac{r^{2}}{R^{2} H_{0 \perp}^{2}}\right\rangle_{D}>3 / 5 .
$$

This spatial domain averaged condition for our LTB void solution can be satisfied in our synchronous comoving gauge with zero vorticity for $\langle\rho\rangle_{D}>0$.

\section{Conclusions}

We have modelled the late-time inhomogeneous and non-linear regime for $z<10$ by an exact spherically symmetric, inhomogeneous solution of Einstein's field equations. The solution approaches an Einstein-de Sitter matter dominated solution for $z>10$, which describes a homogeneous and isotropic FLRW background spacetime with small inhomogeneities $\delta \rho / \rho \sim 10^{-5}$ near the surface of last scattering in agreement with the WMAP data 3. A volume averaging of physical quantities is necessitated for our inhomogeneous universe, and due to the lack of commutativity of the time evolution of the average of the scalar expansion $\theta$, we find that it is possible to have a negative averaged deceleration parameter $\langle q\rangle$ for a positive density of matter $\rho$ and a zero vorticity $\omega=0$. Observational bounds on the magnitude of the quantity $\left\langle P_{D}\right\rangle$ in the condition (57) must be obtained to establish whether a sufficiently negative $q$ can be produced by the expanding inhomogeneous enhancement or void to allow an explanation of the accelerating universe without a negative pressure fluid or a cosmological constant. 
The inhomogeneous solution is exact, so that we do not have to be concerned with the failure of perturbative backreaction calculations in the non-linear latetime universe regime. However, we have assumed a high degree of symmetry for our inhomogeneous solution, since it only allows for a one-dimensional inhomogeneity in the radial direction, although the angular azimuthal expansion described by $H_{\perp}$ does play a significant role in determining the cosmological solution. It would be interesting to investigate inhomogeneous cosmological solutions that possess less symmetry than the LTB models [13].

\section{Acknowledgments}

This work was supported by the Natural Sciences and Engineering Research Council of Canada. I thank Joel Brownstein and Martin Green for helpful discussions.

\section{References}

[1] J. W. Moffat, Jour. Cosmol. Astrophys. JCAP 0510 (2005) 012, astro-ph/0502110

[2] J. W. Moffat, astro-ph/0505326.

[3] C. L. Bennett et al., Ap. J. Suppl. 148, 1 (2003), astro-ph/0302207 D. N. Spergel et al., Ap. J. Suppl. 148. 175 (2003), astro-ph/0302209

[4] S. Perlmutter et al. Ap. J. 517, 565 (1999); A. G. Riess, et al. Astron. J. 116, 1009 (1998); P. M. Garnavich, et al. Ap. J. 509, 74 (1998).

[5] A. G. Riess, et al., Ap. J. 607, 665 (2004), astro-ph/0402512.

[6] F. Hoyle and M. S. Vogeley, Ap. J. 607, 751 (2004), astro-ph/0312533

[7] G. Lemaitre, Mon. Not. Roy. Astron. Soc. 91, 490 (1931); Ann. Soc. Sci. Bruxelles, A53, 51 (1933).

[8] R. C. Tolman, Proc. Nat. Acad. Sci. 20, 169 (1934).

[9] H. Bondi, Mon. Not. Roy. Astron. Soc. 107, 410 (1947).

[10] W. B. Bonnor, Mon. Not. Roy. Astron. Soc. 167, 55 (1974).

[11] J. W. Moffat and D. C. Tatarski, Phys. Rev. D45, 3512 (1992).

[12] J. W. Moffat and D. C. Tatarski, Ap. J. 453, 17 (1995).

[13] For a review of inhomogeneous cosmology and references, see: A Krasiński, Inhomogeneous Cosmological Models, Cambridge University Press, 1997. 
[14] A. Raychaudhuri, Phys. Rev. 98, 1123 (1955).

[15] G. F. R. Ellis, General Relativity and Gravitation (Reidel Pub., Dordrecht), p. 215 (1984).

[16] T. Buchert, G. R. G. 32, 105 (2000), gr-qc/9906015 T. Buchert, Class. Quant. Grav. 22, L113 (2005), gr-qc/0507028.

[17] E. W Kolb, S. Mataresse and A. Riotto, astro-ph/0506534 astro-ph/0511073. 